\title{
Pretreatment central quality control for craniospinal irradiation in non-metastatic medulloblastoma
}

\author{
First experiences of the German radiotherapy quality control panel in the SIOP PNET5 MB trial
}

\begin{abstract}
Stefan Dietzsch ${ }^{1}$ (D) A Annett Braesigk ${ }^{1} \cdot$ Clemens Seidel $^{1} \cdot$ Julia Remmele ${ }^{1} \cdot$ Ralf Kitzing $^{1} \cdot$ Tina Schlender $^{1}$. Martin Mynarek ${ }^{2}$ - Dirk Geismar ${ }^{3} \cdot$ Karolina Jablonska $^{4} \cdot$ Rudolf Schwarz $^{5}$. Montserrat Pazos ${ }^{6} \cdot$ Marc Walser $^{7}$. Silke Frick ${ }^{8}$ Kristin Gurtner ${ }^{9}$. Christiane Matuschek ${ }^{10}$ • Semi Ben Harrabi ${ }^{11}$ • Albrecht Glück ${ }^{12}$ - Victor Lewitzki ${ }^{13}$. Karin Dieckmann ${ }^{14}$ • Martin Benesch ${ }^{15}$ • Nicolas U. Gerber ${ }^{16}$. Stefan Rutkowski ${ }^{2}$ Beate Timmermann ${ }^{3}$. Rolf-Dieter Kortmann'
\end{abstract}

Received: 24 June 2020 / Accepted: 23 October 2020 / Published online: 23 November 2020

(c) The Author(s) 2020

\begin{abstract}
Purpose Several studies have demonstrated the negative impact of radiotherapy protocol deviations on tumor control in medulloblastoma. In the SIOP PNET5 MB trial, a pretreatment radiotherapy quality control (RT-QC) program was introduced. A first analysis for patients enrolled in Germany, Switzerland and Austria with focus on types of deviations in the initial plan proposals and review criteria for modern radiation technologies was performed.
\end{abstract}

Beate Timmermann and Rolf-Dieter Kortmann share last authorship.

Data sharing statement Research data are stored in an institutional repository and will be shared upon request to the corresponding author.

Electronic supplementary material The online version of this article (https://doi.org/10.1007/s00066-020-01707-8) contains supplementary material, which is available to authorized users.

$\triangle$ Dr. med. Stefan Dietzsch, MD

stefan.dietzsch@medizin.uni-leipzig.de

1 Department for Radiation Oncology, University of Leipzig Medical Center, Stephanstr. 9a, 04103 Leipzig, Germany

2 Departement of Pediatric Hematology and Oncology, University Medical Center Hamburg-Eppendorf, Hamburg, Germany

3 Clinic for Particle Therapy, West German Proton Therapy Centre, University of Essen, Essen, Germany

4 Faculty of Medicine, Department of Radiation Oncology, University of Cologne, Cologne, Germany

5 Department of Radiation Oncology, University Medical Center Hamburg-Eppendorf, Hamburg, Germany

6 Department of Radiotherapy and Radiation Oncology, Ludwig Maximilian University Munich, Munich, Germany
7 Center for Protontherapy, Paul Scherrer Institute, Villigen, Switzerland

8 Department of Radiotherapy and Radiation Oncology, Hospital Bremen Mitte, Bremen, Germany

9 Department of Radiotherapy and Radiation Oncology, Faculty of Medicine and University Hospital Carl Gustav Carus, Technical University Dresden, Dresden, Germany

10 Department of Radiation Oncology, Medical Faculty Heinrich Heine University Duesseldorf, Duesseldorf, Germany

11 Department of Radiation Oncology and Radiotherapy, Heidelberg University Hospital, Heidelberg, Germany

12 Radiation Oncology, Munich-Schwabing Municipal Hospital, Munich, Germany

13 Department of Radiotherapy, University of Wuerzburg, Wuerzburg, Germany

14 Department of Radiotherapy, Medical University of Vienna, Vienna, Austria

15 Division of Pediatric Hematology/Oncology, Department of Pediatrics and Adolescent Medicine, Medical University of Graz, Graz, Austria

16 University Children's Hospital of Zurich, Zurich, Switzerland 
Methods and patients Sixty-nine craniospinal irradiation (CSI) plans were available for detailed analyses. RT-QC was performed according to protocol definitions on dose uniformity. Because of the lack of definitions for high-precision 3D conformal radiotherapy within the protocol, additional criteria for RT-QC on delineation and coverage of clinical target volume (CTV) and planning target volume (PTV) were defined and evaluated.

Results Target volume (CTV/PTV) deviations occurred in $49.3 \%$ of initial CSI plan proposals $(33.3 \% \mathrm{minor}$, $15.9 \%$ major). Dose uniformity deviations were less frequent (43.5\%). Modification of the RT plan was recommended in $43.5 \%$ of CSI plans. Unacceptable RT plans were predominantly related to incorrect target delineation rather than dose uniformity. Unacceptable plans were negatively correlated to the number of enrolled patients per institution with a cutoff of 5 patients $(p=0.001)$.

Conclusion This prospective pretreatment individual case review study revealed a high rate of deviations and emphasizes the strong need of pretreatment RT-QC in clinical trials for medulloblastoma. Furthermore, the experiences point out the necessity of new RT-QC criteria for high-precision CSI techniques.

Keywords Quality assurance · Deviation · Brain tumor · Pediatric · Review criteria

\section{Introduction}

In standard risk (SR) medulloblastoma, craniospinal irradiation (CSI) followed by a tumor bed boost is considered the standard treatment in non-infant age groups. Retrospective reports showed that inadequate treatment fields had a negative impact on tumor control and outcome [1-3]. This was confirmed in similar clinical settings [4-6]. Thus, there is a broad consensus that pretreatment quality control of radiotherapy plans (RT-QC) is an important measure in multiinstitutional clinical trials [7-10].

In 2014, the European branch of the International Society of Pediatric Oncology (SIOPe) initiated the SIOP PNET5 MB trial for children with non-metastatic medulloblastoma with a low-risk or average-risk biological profile (ClinicalTrials.gov identifier: NCT02066220). The quality assurance (QA) program included a central review of pathology, magnetic resonance imaging (MRI), and radiotherapy (RT). Furthermore, pretreatment RT-QC was considered a mandatory component of the study. This report describes a first analysis for patients enrolled in Germany, Austria, and Switzerland, with focus on the type of deviations in the initial plan proposals and review criteria for modern radiation technologies.

\section{Materials and methods}

SIOP PNET5 MB is a prospective phase II/III study in patients between the ages of 3 to 5 years and 21 years with clinical SR medulloblastomas according to the risk group definitions which have been used so far, e.g., in HIT-SIOP PNET4 [11-13]. Patients with low-risk biological profile (PNET5 MB, LR arm) received 18Gy to the craniospinal
Fig. 1 Workflow and time schedule for quality control from surgery to start of radiotherapy. MRI Magnetic resonance imaging, $C S F$ cerebrospinal fluid, $R T$ radiotherapy, $R T-Q C$ quality control of radiotherapy

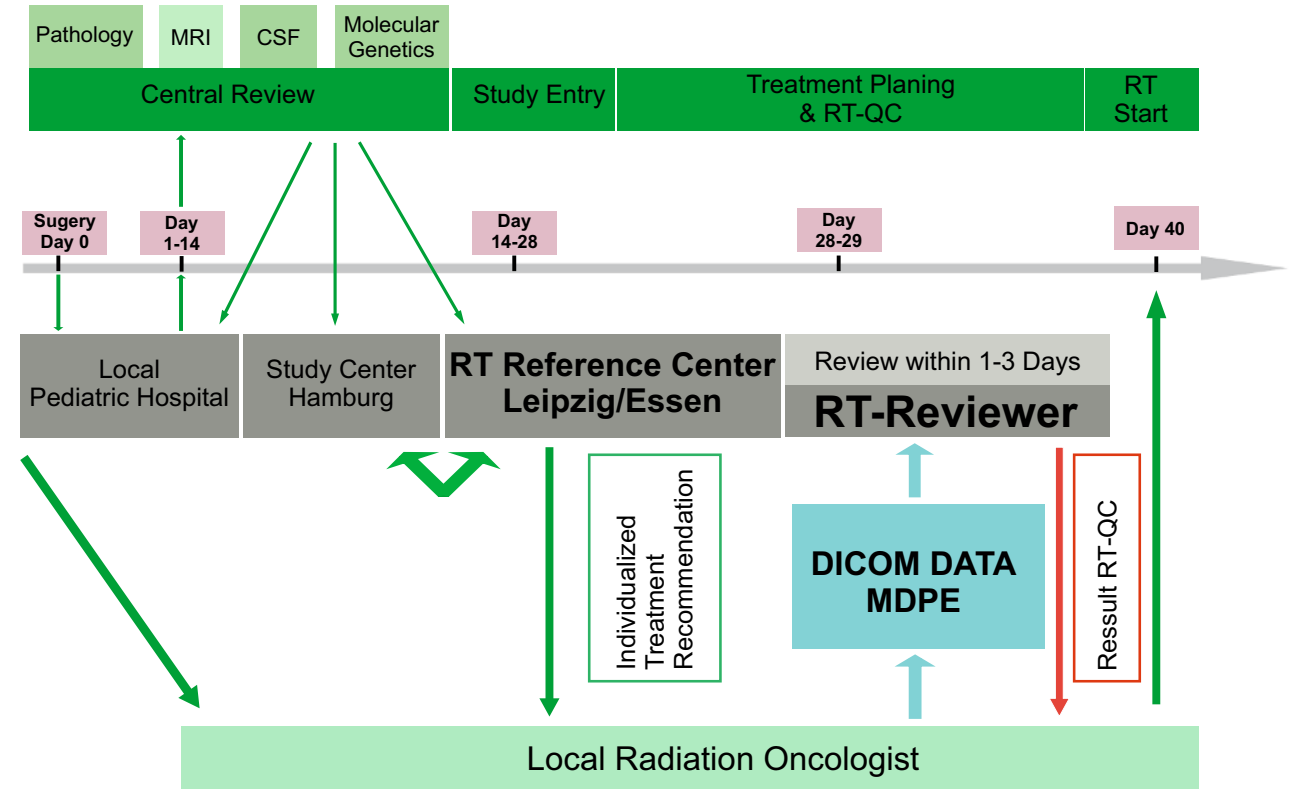


Table 1 SIOP PNET5 protocol definitions of radiotherapy parameters for quality control and additional definitions of target delineation as minor or major deviation of the reference center

\begin{tabular}{|c|c|c|c|}
\hline & Per protocol & Minor deviation & Major deviation \\
\hline \multicolumn{4}{|c|}{ Protocol definitions } \\
\hline \multicolumn{4}{|c|}{ Target volume } \\
\hline \multicolumn{4}{|c|}{ CSI (distance from field edge to CTV) } \\
\hline $\begin{array}{l}\text { Cribriform } \\
\text { plate }\end{array}$ & $\geq 5 \mathrm{~mm}$ & 3 to $<5 \mathrm{~mm}$ & $<3 \mathrm{~mm}$ \\
\hline $\begin{array}{l}\text { All other } \\
\text { regions }\end{array}$ & $\geq 10 \mathrm{~mm}$ & 5 to $<10 \mathrm{~mm}$ & $<5 \mathrm{~mm}$ \\
\hline \multicolumn{4}{|c|}{ Dose uniformity } \\
\hline V95\% & $\geq 95 \%$ & $\geq 90$ to $<95 \%$ & $<90 \%$ \\
\hline V107\% & $\leq 5 \%$ & $>5 \%$ to $<10 \%$ & $\geq 10 \%$ \\
\hline
\end{tabular}

Additional definitions by the reference center

Target volume delineation

\begin{tabular}{llll} 
CTV/PTV & $\begin{array}{l}\text { Entire subarachnoid space encom- } \\
\text { passed by PTV and CTV }\end{array}$ & $\begin{array}{l}\text { Entire subarachnoid space not encom- } \\
\text { passed by CTV but by PTV }\end{array}$ & $\begin{array}{l}\text { Entire subarachnoid space not encom- } \\
\text { passed by CTV and PTV }\end{array}$ \\
\hline
\end{tabular}

CSI Craniospinal irradiation, CTV clinical target volume, PTV planning target volume, V95\% proportion of PTV which is covered by the $95 \%$ isodose, V107\% proportion of PTV which receive $\geq 107 \%$ of the prescribed dose

axis. All other SR patients (PNET5 MB, SR arm) received 23.4 Gy CSI. The tumor site was boosted up to 54 Gy. Patients recruited to the SIOP PNET5 MB SR and LR strata until December 31, 2018 in Germany, Switzerland and Austria and with complete available DICOM-RT data of CSI plans were considered eligible for this report.

RT-QC was performed by the Reference Center for Radiotherapy in Childhood Brain Tumors in Leipzig in collaboration with the West German Proton Center in Essen. Workflow and time schedule of RT-QC are shown in Fig. 1. A benchmark case or dummy run was not performed. All local radiation oncologists received a detailed individualized treatment recommendation including target delineation and dosimetric aims. Central plan analyses were performed by importing the original plan data including dose files into the local treatment planning systems of the reference centers (RayStation, Raysearch Laboratories, Stockholm, Sweden). CSI techniques were documented and grouped into three categories: 3D conformal (lateral opposing fields for brain and posterior fields for spine), high precision photon (intensity-modulated radiotherapy with fixed gantry angles [IMRT], volumetric modulated arc therapy [VMAT], tomotherapy), or proton beam therapy (with active scanning). Typical dose distributions are available in the supplementary material (Fig. S1). In order to analyze possible changes during accrual, time of enrollment was divided in two periods. Cut-off was the median of enrolled patients at the end of 2016.

For SIOP PNET5 MB RT-QC, minor and major deviations of dose uniformity were defined according to ICRU 50/62 (Table 1; $[14,15]$ ). Target deviations were used according to Carrie et al. [1]. These definitions address the distance from the field edge to the clinical target volume (CTV) and are therefore only suitable for simulation-based treatment planning and 3D conformal techniques with opposing lateral fields of the brain and posterior fields of the spinal axis. For high-precision CSI techniques, RTQC adaptations of the former concept described by Carrie et al. were performed in order to better evaluate the CTV/PTV and their coverage by the $95 \%$ isodose, with a special focus on the critical areas cribriform plate, temporal lobe, and thecal sac (Table 1; [1]). Since 2018, CTV has been evaluated according to the SIOP guideline on craniospinal target volume delineation with additional special focus on skull base foramina [16]. The SIOP PNET5 MB protocol required contouring of organs at risk (OAR) and collection of corresponding dose exposure. $30 \mathrm{~Gy}$ to at least one cochlea is the only dose constraint to an OAR. Contouring and dose to OAR was not a criterion for plan acceptance.

Furthermore, deviations were checked for clinical relevance (expected increased risk for relapse and/or increased risk for toxicity) and scored as "acceptable" or "unacceptable" at the discretion of the reviewer and based on the experience of treatment plan evaluation in the SIOP Neuroblastoma protocol [17]. The overall QA result was rated as "per protocol," "acceptable deviation," or "unacceptable deviation." If modifications were required, results of RTQC were communicated to the treating institution by telephone and/or Email including illustrating screenshots. The final result was subsequently communicated by a formal letter.

Associations between variables were examined using $\chi^{2}$ tests. All statistical analyses were performed using the Statistical Package for Social Sciences (IBM SPSS statistics), version 24 (IBM, Armonk, NY, USA). 
Fig. 2 Influence of experience with protocol on quality control result. $n$ depicts number of patients who were evaluated with respect to the patients in radiotherapy unit (e.g., 24 patients were evaluated as the first patient in an unit). $R T$ Radiotherapy

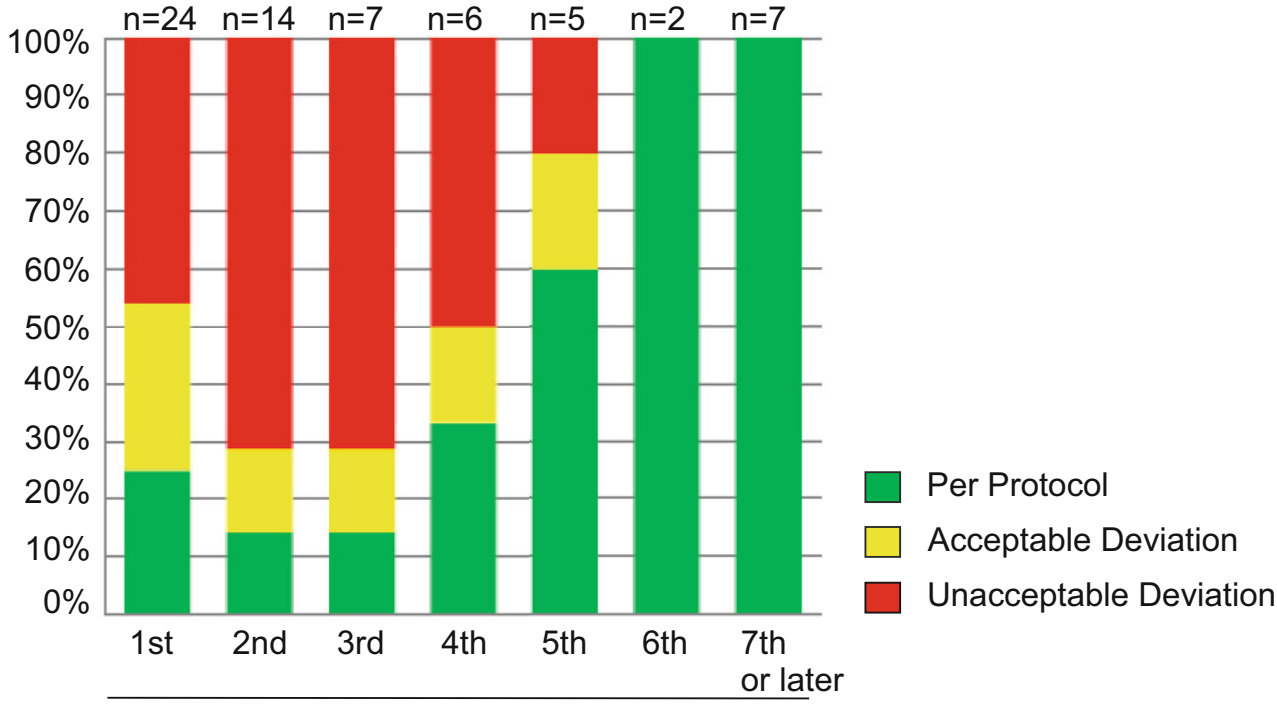

Patient in RT Unit

\section{Results}

Between September 2014 and December 2018, 70 German, 6 Swiss, and 2 Austrian patients were enrolled in the SIOP PNET5-MB trial and treated in 29 institutions. In 8 patients, no DICOM-RT data were available. One patient was treated by a simulation-based treatment technique. In this patient, modification of RT portals at the cribriform plate was recommended. The patient was excluded from analysis because of the lack of CTV/PTV structures and dose uniformity data. Sixty-nine CSI plans were available for detailed evaluation. Forty-six patients $(66.6 \%)$ were treated in the SR arm and 23 patients (33.3\%) in the LR arm. Supplementary Table $1 \mathrm{~S}$ summarizes the frequency of different CSI techniques over the evaluated period. The data indicate a shift from 3D conformal techniques being the most common between 2014 and 2016, to proton therapy being the most common to date.

Supplementary Table S2 summarizes the frequency of target volume and dose uniformity deviations in the initial plan proposals. Target volume deviations occurred in $49.3 \%$ of RT plans and were more frequent in the brain CTV/PTV $(30.4 \%)$ than in the spinal CTV/PTV $(24.6 \%)$. Dose uniformity deviations were found in $43.5 \%$ of the RT plans and were more frequent in the spinal PTV. The frequency of dose uniformity deviation was higher for 3D conformal radiotherapy (72.7\% major, $22.7 \%$ minor) than for highprecision techniques, in which $80.9 \%$ were per protocol $\left(\chi^{2}\right.$ $p<0.001$ ). Only 1 of $223 \mathrm{D}$ conformal radiotherapy plans met the dose uniformity criteria of the protocol. For highprecision techniques, the median V95 (proportion of PTV which is covered by the $95 \%$ isodose) was $99.1 \% \pm 2 \%$ for PTV-brain and $98.3 \% \pm 5.2 \%$ for PTV-spine. V107 (pro- portion of PTV which receives $\geq 107 \%$ of the prescribed dose) was median $0 \%$ for both PTVs.

Only 27 plans (39.1\%) did not have any deviation. In $17.4 \%(n=12)$ of the RT plans, deviations were considered as acceptable and in $30(43.5 \%)$ plan modifications were recommended. The rate of recommended CSI plan modifications was higher in low-recruiting ( $\leq 4$ patients) than in high-recruiting ( $\geq 5$ patients) radiotherapy units $(62.1 \%$ vs. $\left.30 \% ; \chi^{2} p=0.005\right)$. An impact of institutional experience in treating SIOP PNET5 MB trial patients on the rate of unaccepted RT plans was also observed (Fig. 2). High rates of unacceptable deviations were seen in the first 4 patients in all RT units. The rate declined when enrolling the fifth and following patients (first to fourth patient in unit $56.9 \%$ vs. fifth or later patient in unit $5.6 \% ; \chi^{2} p<0.001$ ). The percentage of recommended CSI plan modifications did not decrease over time and was $44.1 \%$ in $2014-2016$ and $42.9 \%$ in $2017-2018\left(\chi^{2} p=0.916\right)$.

Table 2 shows the interaction between the type of deviation and requirement of RT plan modification. Only 1 RT plan with dose uniformity deviation alone required plan modification. In 9 of $30(30 \%)$ cases with unacceptable deviations, only target volume deviations were present, with a correct assessment of dose uniformity. 20 of 30 unacceptable plans showed both types of deviation.

\section{Discussion}

The present study represents a large cohort of a pretreatment, fully digital individual case review RT-QC procedure in medulloblastomas treated by CSI. 
Table 2 Interaction between target and dose uniformity deviations with respect to overall result (acceptable/unacceptable) of the initial plan proposals of the local radiation oncologist

\begin{tabular}{|c|c|c|c|c|}
\hline & Per protocol & Acceptable deviation & Unacceptable deviation & Total \\
\hline Correct target and dose & 27 & 0 & 0 & 27 \\
\hline $\begin{array}{l}\text { Correct target but deviation dose } \\
\text { uniformity }\end{array}$ & 0 & $\begin{array}{l}7 \\
\text { dose minor } 3 \\
\text { dose major } 4\end{array}$ & $\begin{array}{l}1 \\
\text { dose minor } 1\end{array}$ & 8 \\
\hline $\begin{array}{l}\text { Deviation target but correct dose } \\
\text { uniformity }\end{array}$ & 0 & $\begin{array}{l}3 \\
\text { target minor } 3\end{array}$ & $\begin{array}{l}9 \\
\text { target minor } 6 \\
\text { target major } 3\end{array}$ & 12 \\
\hline Deviation target and dose uniformity & 0 & $\begin{array}{l}2 \\
\text { target minor } 2 \\
\text { dose major } 2\end{array}$ & $\begin{array}{l}20 \\
\text { target minor } 12 \\
\text { target major } 8 \\
\text { dose minor } 5 \\
\text { dose major } 15\end{array}$ & 22 \\
\hline Total & 27 & 12 & 30 & 69 \\
\hline
\end{tabular}

\section{Observed deviations}

We observed a high rate of RT protocol deviations in the initial plan proposals (49.3\% target delineation, $43.5 \%$ dose uniformity). 30 of 69 (43.5\%) RT plans were clinically unacceptable and modifications were recommended. High rates of RT protocol deviations were also reported in other medulloblastoma trials. Most of these retrospective studies were based on the evaluation of simulation films of lateral opposing fields for the brain and posterior fields for the spinal canal [1, 2, 18, 19]. First experiences of pretreatment central RT-QC of SIOP PNET5 MB patients treated in Italy revealed a necessity of plan revision in $62.5 \%$ of CSI plans. Most common reasons were target delineation and covering of the cribriform plate or the thecal sac [20].

\section{CTV/PTV delineation}

Definitions of targeting deviations in the SIOP PNET5 MB protocol adopted the QC process of the HIT-SIOP PNET4 trial or Carrie et al., respectively [1, 12]. This included the distance from the field edge and bony reference structures to the CTV in lateral opposing fields for the brain and dorsal fields for the spinal canal. The use of this technique, however, decreased from approximately 50\% in 2014-2016 to $14 \%$ in 2017-2018. Modern high-precision technologies (IMRT, VMAT, tomotherapy, and in particular proton beam therapy) were increasingly used to decrease the risk of late toxicity and can be considered as standard of care today [21-24]. Presently, there is no established standardized definition of CSI targeting deviations for high-precision CSI techniques available. However, both review of target delineation and assessment of dose uniformity are indispensable for evaluating a treatment plan for any risk of relapse. This necessity is emphasized by our findings that target deviations were more common than dose uniformity deviations in the 30 plans considered as "unacceptable" $(3.3 \%$ unacceptable dose uniformity alone, $30 \%$ unacceptable target definition alone, $66.7 \%$ both deviations). Similar results were observed in the Italian cohort [20]. Moreover, 9 of our 69 plans $(13 \%)$ or 9 of 30 unacceptable deviations $(30 \%)$ would have been scored as acceptable based on the dose uniformity criteria alone defined in the protocol.

\section{Dose uniformity and regional dose distribution}

Evaluation of dose uniformity deviations has three short comings. In case of posterior treatment fields, major deviations can occur due to a formal underdosage in anterior parts or overdosage in posterior parts of the spinal PTV. This can especially be the case when the whole vertebral bodies are included into the spinal PTV to prevent a dose gradient $>5$ Gy or $>70 \%$ within the vertebral bodies [25]. These deviations, when caused by radiation techniques but not due to planning inadequacies, were judged as acceptable deviations not requiring any modification.

The V95\% constraint has to be used with caution in the case of large PTVs. The volume of PTV-brain varies depending on age and is more than $1000 \mathrm{~cm}^{3}$. The constraint V95\% $\geq 95 \%$ for a PTV of $1000 \mathrm{~cm}^{3}$ means that underdosages in $50 \mathrm{~cm}^{3}$ will still be considered as per protocol. The cribriform plate has been demonstrated to be a critical region for relapse in medulloblastoma [1, 3, 26]. Because of the small volume circumscribed, marked underdosage can occur, although formally, a correct dose uniformity was calculated. Therefore, regional dose distribution has to be evaluated, especially in critical regions like the cribriform plate or temporal lobes (Fig. 3).

Furthermore, the circumscribed regional dose distribution should be evaluated in regions of inadequate CTV and PTV delineation. In our cohort, five cases of inadequate CTV were identified not including the skull base foramina. But for technical reasons, this region was encompassed by the $95 \%$ isodose. Therefore, this major targeting deviation 

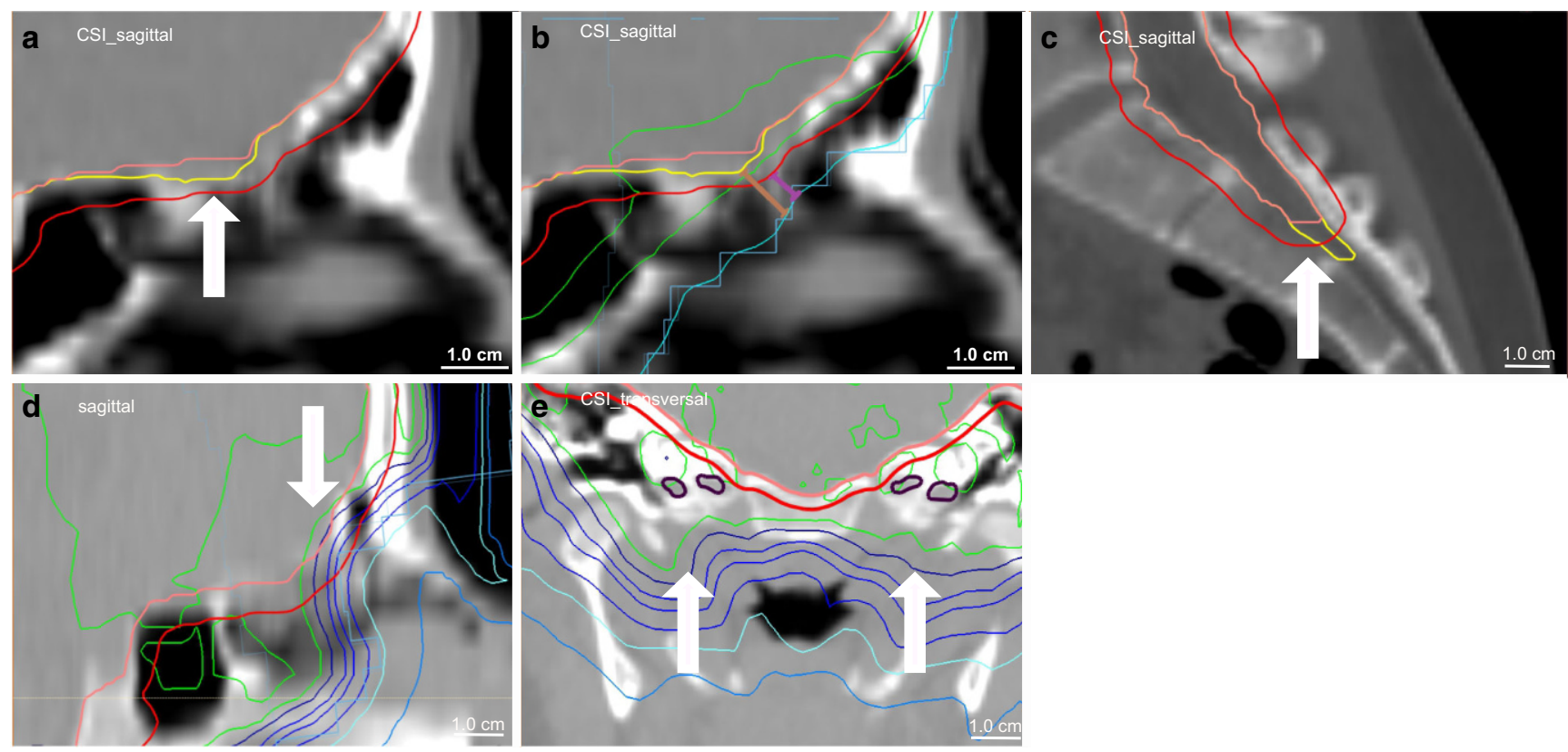

Fig. 3 Examples of proposed deviations. a Acceptable deviation - the subarachnoid space according SIOP guideline (yellow)[16] is not encompassed by CTV (pink) but by the PTV (red). b Background for acceptable deviation in (a) - the distance from field edge (leaf) and $50 \%$ isodose (light blue, as equivalent to a customized block) to the PTV (red) is $5 \mathrm{~mm}$ (purple) and to the subarachnoid space (correct reference CTV; yellow) approximately $8 \mathrm{~mm}$ (brown), which is per protocol according to the SIOP PNET5 protocol definitions in Table 1. The 95\% isodose does not completely cover the PTV (red) but the subarachnoid space (yellow). c Major/unacceptable deviation—the subarachnoid space (thecal sac) is not encompassed by CTV (pink) and PTV (red). d Unacceptable circumscribed regional dose in case of correct target definition and dose uniformity - the subarachnoid space (cribriform plate) is encompassed by CTV (pink) and PTV (red) but the 95\% isodose does not cover this critical region because of eye-sparing plan optimization. e Acceptable plan in case of unacceptable target volume deviation-the subarachnoid space (scull base foramina; violet) is not encompassed by CTV (pink) and PTV (red) but is covered by the 95\% isodose (outer light green line) due to radiation technique. SIOP International Society of Paediatric Oncology, CTV clinical target volume, PTV planning target volume, CSI craniospinal irradiation

was judged as acceptable without requiring any modification (Fig. 3).

As a consequence, major deviations were not automatically unacceptable. On the other hand, a plan with formally correct dose uniformity could be unacceptable, e.g., because of inadequate CTV delineation or dose coverage at critical regions. Due to these observations, it seems to be necessary to renew the criteria for CSI-QC and to define acceptable and unacceptable deviations.

\section{New QC criteria}

From our experience, we would propose defining different criteria for 3D conformal plans (lateral opposing fields for brain and posterior fields for spine) and high-precision CSI techniques. QC of 3D conformal plans can be based on the criteria of Carrie et al. [1]. The dose uniformity criteria can be used for PTV-brain but seem to be unsuitable for the spinal PTV when covered by posterior spinal fields. For high-precision techniques, we propose the new criteria shown in Table 3 and illustrated in Fig. 3, which include CTV/PTV contouring, circumscribed regional dose, vertebral dose in children before the end of the pubertal acceleration phase [25], and dose uniformity. The basis of RT-QC criteria is dose coverage of the entire subarachnoid space defined according to the SIOP contouring guideline [16]. CTV delineation according to this guideline is strongly recommended. The criteria for CTV/PTV delineation adopt the margins between field edge and CTV of Carrie et al. [1] and the SIOP PNET 4 protocol [12]. Due to the typical lateral dose gradient in photon therapy, approximately $50 \%$ of the prescribed dose is given at the field edge and $95 \%$ of dose is applied approximately 3 to $5 \mathrm{~mm}$ distant to the field edge within the target. Furthermore, we find adequate dose uniformity in the majority of cases when highprecision CSI techniques were used. Therefore, we propose using a stricter dose uniformity constraint (V95\% $\geq 98 \%)$ as was recommended by the ICRU 83 report for IMRT techniques [27]. The same constraints should also be used for proton plans, respecting the recommendations of the ICRU 78 report [28]. 
Table 3 Proposal for definitions of acceptable and unacceptable deviations and final result of quality control in craniospinal irradiation with high-precision techniques

\begin{tabular}{|c|c|c|}
\hline & Acceptable deviation & Unacceptable deviation \\
\hline \multicolumn{3}{|c|}{ Target volume delineation } \\
\hline CTV/PTV & $\begin{array}{l}\text { Entire subarachnoid space }{ }^{\mathrm{a}} \text { not encompassed by } C T V \\
\text { but by PTV } \\
\text { or } \\
\text { PTV larger than necessary } \\
\left(\leq 0.5 \mathrm{~cm} \text { areas with effect on OAR dose }{ }^{\mathrm{b}} \leq 1.0 \mathrm{~cm}\right. \\
\left.\text { areas without effect on OAR dose }{ }^{\mathrm{b}}\right)\end{array}$ & $\begin{array}{l}\text { Entire subarachnoid space }{ }^{\mathrm{a}} \text { not encompassed by } C T V \text { and } \\
P T V \\
\text { or } \\
\text { PTV substantially larger than necessary } \\
\left(>0.5 \mathrm{~cm} \text { areas with effect on OAR dose } \mathrm{e}^{\mathrm{b}}>1.0 \mathrm{~cm} \text { areas }\right. \\
\left.\text { without effect on OAR dose } \mathrm{e}^{\mathrm{b}}\right)\end{array}$ \\
\hline \multicolumn{3}{|c|}{ Circumscribed regional dose coverage at critical locations (cribriform plate, temporal lobes, skull base foramina, thecal sac) } \\
\hline 95\%-isodose & Not defined & Does not encompass entire subarachnoid space ${ }^{a}$ \\
\hline \multicolumn{3}{|c|}{ Vertebral dose in children before end of pubertal acceleration phase } \\
\hline $\begin{array}{l}\text { Anterior-posterior dose } \\
\text { gradient }\end{array}$ & Not defined & $>5 \mathrm{~Gy}$ or $70 \%$ \\
\hline \multicolumn{3}{|l|}{ Dose uniformity } \\
\hline$V 95 \%$ & $\geq 95$ to $<98 \%$ & $<95 \%$ \\
\hline V107\% & $>5 \%$ to $<10 \%$ & $\geq 10 \%$ \\
\hline \multicolumn{3}{|l|}{ Final result of RT-QC } \\
\hline \multicolumn{3}{|l|}{ Per protocol } \\
\hline \multicolumn{3}{|c|}{$\begin{array}{l}\text { Acceptable deviation } \\
\text { (in individual cases, when missing part of target volume is covered by dose due to radiation technique, final result can be acceptable deviation } \\
\text { even in case of unacceptable target volume deviation) }\end{array}$} \\
\hline \multicolumn{3}{|l|}{ Unacceptable deviation } \\
\hline
\end{tabular}

CTV Clinical target volume, PTV planning target volume, OAR organ at risk, V95\% proportion of PTV which is covered by the $95 \%$ isodose, $V 107 \%$ proportion of PTV which receive $\geq 107 \%$ of the prescribed dose, $R T-Q C$ quality control of radiotherapy, SIOP International Society of Paediatric Oncology

${ }^{a}$ Correct reference CTV according SIOP guideline [16]

${ }^{\mathrm{b}}$ For example, eye globe, lens, thyroid, lung, heart, esophagus, kidney

${ }^{c}$ according SIOP consensus recommendations [25]

\section{Impact of experience with RT guidelines of the protocol}

The rate of unaccepted RT plans in our cohort depends on the experience of the radiotherapy units in treating SIOP PNET5 MB patients, with a cutoff of 5 patients per institution. A comparable impact on the institution's familiarity with the protocol was seen in RT-QC of meningioma in the EORTC 22042-26042 trial [6]. Fewer deviations were observed in RT plans from high-recruiting institutions ( $\geq 5$ patients) compared to those from low-recruiting centers ( $22 \%$ vs. $62 \%, p=0.007)$. The recently published Italian experience of SIOP PNET5 MB RT-QC also showed a higher rate of corrected major deviations in patients when treated in less experienced centers (88.2\%) compared to the whole cohort $(62.5 \%)$ [20]. Interestingly, a decrease in unacceptable RT plans over time was not observed. This could partly be explained by the continuous high percentage of patients included by low-recruiting radiotherapy units ( $\leq 4$ patients; $47.1 \%$ in $2014-2016$ and $37.1 \%$ in $2017-2018$; $p=0.404$ ). Additionally, the number of new institutions starting recruitment of patients (patient 1 to 4 ) remained high in the second period $(57.1 \%)$.
Our observations underline the fundamental role of upfront RT-QC to ensure protocol-compliant RT and confirmed the findings of the M-SFOP 98 protocol. In this trial, craniospinal fields were reviewed before the start of radiotherapy. Major deviations were observed in 14 of 55 patients; 9 of these deviations were due to eye shielding and 8 of them were modified before RT start [29]. It remains to be seen how far the recently published European guideline for craniospinal CTV contouring will help to improve target delineation [16]. Furthermore, a training program for local radiation oncologists including benchmark cases and with a final certification of investigators has to be considered.

\section{Conclusion}

This pretreatment individual case review study revealed a high rate of protocol deviations and emphasizes the strong need for pretreatment RT-QC in clinical trials on medulloblastoma, particularly in low-recruiting centers and for approximately the first 5 patients of each RT institution. Therefore, pretreatment QA programs are desirable to support decentralized treatment in multicenter trials. Moreover, 
our experiences point out the necessity of new RT-QC criteria for high-precision CSI techniques.

Acknowledgements The authors thank all attending physicians and physicists of the participating centers for their cooperation and the Deutsche Kinderkrebsstiftung (German Childhood Cancer Foundation) for its support.

Funding The analysis was supported by the German Childhood Cancer Foundation (Deutsche Kinderkrebsstiftung).

Funding Open Access funding enabled and organized by Projekt DEAL.

\section{Compliance with ethical guidelines}

Conflict of interest S. Dietzsch reports grants from the German Childhood Cancer Foundation (Deutsche Kinderkrebsstiftung; DKS2016.01A) during the conduct of the study. A. Braesigk reports grants from the German Childhood Cancer Foundation (Deutsche Kinderkrebsstiftung; DKS2014.18) during the conduct of the study. T. Schlender reports grants from the German Childhood Cancer Foundation (Deutsche Kinderkrebsstiftung; DKS2018.04) during the conduct of the study. M. Mynarek reports grants from the German Childhood Cancer Foundation (Deutsche Kinderkrebsstiftung; DKS2013.15) during the conduct of the study. S. Rutkowski reports grants from German Childhood Cancer Foundation (Deutsche Kinderkrebsstiftung; DKS2013.15) during the conduct of the study. C. Seidel, J. Remmele, R. Kitzing, D. Geismar, K. Jablonska, R. Schwarz, M. Pazos, M. Walser, S. Frick, K. Gurtner, C. Matuschek, S.B. Harrabi, A. Glück, V. Lewitzki, K. Dieckmann, M. Benesch, N.U. Gerber, B. Timmermann, and R.-D. Kortmann declare that they have no competing interests.

Ethical standards The SIOP PNET5 MB trial (ClinicalTrials.gov identifier: NCT02066220) was approved by the Ethics Committee Hamburg. Informed consent of the patients or their legal guardian was obtained in all patients.

Open Access This article is licensed under a Creative Commons Attribution 4.0 International License, which permits use, sharing, adaptation, distribution and reproduction in any medium or format, as long as you give appropriate credit to the original author(s) and the source, provide a link to the Creative Commons licence, and indicate if changes were made. The images or other third party material in this article are included in the article's Creative Commons licence, unless indicated otherwise in a credit line to the material. If material is not included in the article's Creative Commons licence and your intended use is not permitted by statutory regulation or exceeds the permitted use, you will need to obtain permission directly from the copyright holder. To view a copy of this licence, visit http://creativecommons.org/licenses/by/4. $0 /$.

\section{References}

1. Carrie C, Hoffstetter S, Gomez F et al (1999) Impact of targeting deviations on outcome in medulloblastoma: study of the French Society of Pediatric Oncology (SFOP). Int J Radiat Oncol Biol Phys 45(2):435-439

2. Kortmann RD, Timmermann B, Kuhl J et al (1999) HIT '91 (prospective, co-operative study for the treatment of malignant brain tumors in childhood): accuracy and acute toxicity of the ir- radiation of the craniospinal axis. Results of the quality assurance program. Strahlenther Onkol 175(4):162-169

3. Miralbell R, Bleher A, Huguenin P et al (1997) Pediatric medulloblastoma: radiation treatment technique and patterns of failure. Int J Radiat Oncol Biol Phys 37(3):523-529

4. Fairchild A, Weber DC, Bar-Deroma R et al (2012) Quality assurance in the EORTC 22033-26033/CE5 phase III randomized trial for low grade glioma: the digital individual case review. Radiother Oncol 103(3):287-292. https://doi.org/10.1016/j.radonc.2012. 04.002

5. Gondi V, Cui Y, Mehta MP et al (2015) Real-time pretreatment review limits unacceptable deviations on a cooperative group radiation therapy technique trial: quality assurance results of RTOG 0933. Int J Radiat Oncol Biol Phys 91(3):564-570. https://doi.org/ 10.1016/j.ijrobp.2014.10.054

6. Coskun M, Straube W, Hurkmans CW et al (2013) Quality assurance of radiotherapy in the ongoing EORTC 22042-26042 trial for atypical and malignant meningioma: results from the dummy runs and prospective individual case reviews. Radiat Oncol 8:23. https:// doi.org/10.1186/1748-717X-8-23

7. Goodman KA (2013) Quality assurance for radiotherapy: a priority for clinical trials. JNCI J Natl Cancer Inst 105(6):376-377. https:// doi.org/10.1093/jnci/djt031

8. Weber DC, Tomsej M, Melidis C et al (2012) QA makes a clinical trial stronger: evidence-based medicine in radiation therapy. Radiother Oncol 105(1):4-8. https://doi.org/10.1016/j.radonc.2012.08. 008

9. FitzGerald TJ (2014) A new model for imaging and radiation therapy quality assurance in the National Clinical Trials Network of the National Cancer Institute. Int $\mathbf{J}$ Radiat Oncol Biol Phys 88(2):272-273. https://doi.org/10.1016/j.ijrobp.2013.09.030

10. Fairchild A, Straube W, Laurie F et al (2013) Does quality of radiation therapy predict outcomes of multicenter cooperative group trials? A literature review. Int J Radiat Oncol Biol Phys 87(2):246-260. https://doi.org/10.1016/j.ijrobp.2013.03.036

11. Bartlett F, Kortmann R, Saran F (2013) Medulloblastoma. Clin Oncol 25(1):36-45. https://doi.org/10.1016/j.clon.2012.09.008

12. Lannering B, Rutkowski S, Doz F et al (2012) Hyperfractionated versus conventional radiotherapy followed by chemotherapy in standard-risk medulloblastoma: results from the randomized multicenter HIT-SIOP PNET 4 trial. J Clin Oncol 30(26):3187-3193. https://doi.org/10.1200/JCO.2011.39.8719

13. Zeltzer PM, Boyett JM, Finlay JL et al (1999) Metastasis stage, adjuvant treatment, and residual tumor are prognostic factors for medulloblastoma in children: conclusions from the Children's Cancer Group 921 randomized phase III study. J Clin Oncol 17(3):832-845. https://doi.org/10.1200/JCO.1999.17.3.832

14. Landberg T, Chavaudra J, Dobbs J et al (2016) Report 50. J ICRU. https://doi.org/10.1093/jicru/os26.1.Report50

15. Landberg T, Chavaudra J, Dobbs J et al (2016) Report 62. J ICRU. https://doi.org/10.1093/jicru/os32.1.Report62

16. Ajithkumar T, Horan G, Padovani L et al (2018) SIOPE-brain tumor group consensus guideline on craniospinal target volume delineation for high-precision radiotherapy. Radiother Oncol 128(2):192-197. https://doi.org/10.1016/j.radonc.2018.04.016

17. Gaze MN, Boterberg T, Dieckmann K et al (2013) Results of a quality assurance review of external beam radiation therapy in the International Society of Paediatric Oncology (Europe) Neuroblastoma Group's high-risk neuroblastoma trial: a SIOPEN study. Int J Radiat Oncol Biol Phys 85(1):170-174. https://doi.org/10.1016/j. ijrobp.2012.05.004

18. Miralbell R, Fitzgerald TJ, Laurie F et al (2006) Radiotherapy in pediatric medulloblastoma: quality assessment of Pediatric Oncology Group trial 9031. Int J Radiat Oncol Biol Phys 64(5):1325-1330. https://doi.org/10.1016/j.ijrobp.2005.11.002 
19. Donahue B, Marymont MAH, Kessel S et al (2012) Radiation therapy quality in CCG/POG intergroup 9961: implications for craniospinal irradiation and the posterior fossa boost in future medulloblastoma trials. Front Oncol 2:185. https://doi.org/10.3389/fonc. 2012.00185

20. Meroni S, Cavatorta C, Barra S et al (2019) Ein spezielles CloudSystem für die Real-time-/Vorab-Qualitätssicherung in der pädiatrischen Strahlentherapie. Strahlenther Onkol. https://doi.org/10. 1007/s00066-019-01469-y (A dedicated cloud system for real-time upfront quality assurance in pediatric radiation therapy)

21. Laprie A, Hu Y, Alapetite C et al (2015) Paediatric brain tumours: a review of radiotherapy, state of the art and challenges for the future regarding protontherapy and carbontherapy. Cancer Radiother 19(8):775-789. https://doi.org/10.1016/j.canrad.2015.05.028

22. Yock TI, Yeap BY, Ebb DH et al (2016) Long-term toxic effects of proton radiotherapy for paediatric medulloblastoma: a phase 2 single-arm study. Lancet Oncol 17(3):287-298. https://doi.org/ 10.1016/S1470-2045(15)00167-9

23. Eaton BR, Esiashvili N, Kim S et al (2016) Endocrine outcomes with proton and photon radiotherapy for standard risk medulloblastoma. Neuro Oncol 18(6):881-887. https://doi.org/10.1093/neuonc/ nov302

24. Huynh M, Marcu LG, Giles E et al (2019) Are further studies needed to justify the use of proton therapy for paediatric cancers of the central nervous system? A review of current evidence. Radiother Oncol 133:140-148. https://doi.org/10.1016/j.radonc.2019.01. 009
25. Hoeben BA, Carrie C, Timmermann B et al (2019) Management of vertebral radiotherapy dose in paediatric patients with cancer: consensus recommendations from the SIOPE radiotherapy working group. Lancet Oncol 20(3):e155-e166. https://doi.org/10.1016/ S1470-2045(19)30034-8

26. Taylor RE, Donachie PHJ, Weston CL et al (2009) Impact of radiotherapy parameters on outcome for patients with supratentorial primitive neuro-ectodermal tumours entered into the SIOP/UKCCSG PNET 3 study. Radiother Oncol 92(1):83-88. https://doi.org/10.1016/j.radonc.2009.02.017

27. The International Commission on Radiation Units and Measurements (2010) Prescribing, recording, and reporting intensity-modulated photon-beam therapy (IMRT). J ICRU. https://doi.org/10. 1093/jicru/ndq001

28. The International Commission on Radiation Units and Measurements (2007) Prescribing, recording and reporting proton-beam therapy. J ICRU. https://doi.org/10.1093/jicru/ndm021

29. Carrie C, Muracciole X, Gomez F et al (2005) Conformal radiotherapy, reduced boost volume, hyperfractionated radiotherapy, and online quality control in standard-risk medulloblastoma without chemotherapy: results of the French M-SFOP 98 protocol. Int J Radiat Oncol Biol Phys 63(3):711-716. https://doi.org/10.1016/j. ijrobp.2005.03.031 